\title{
Lack of accountability in upholding authorship standards in prominent medical oncology clinical trials
}

\author{
D.Y. Gui PhD* and G.J. Weiss MD MBA*十
}

\begin{abstract}
Authorship in biomedical publications is critical for establishing accountability and contribution toward clinical and scientific research. We examined the frequency of discordance in authorship between presentations of clinical trial data at annual meetings of the American Society of Clinical Oncology and the subsequent peer-reviewed publications. We found that more than $70 \%$ of subsequent publications had additional authors not originally present on the abstract despite there being no changes in trial accrual or trial design. This pervasive discordance in authorship demonstrates a lack of uniformity and accountability in authorship reporting standards.
\end{abstract}

Key Words Clinical trials; authorship, honorary

Curr Oncol. 2019 October;26(5):e693-e695

www.current-oncology.com

\section{INTRODUCTION}

To establish consistent standards for authorship, the International Committee of Medical Journal Editors (ICMJE) developed guidelines for authorship ${ }^{1}$. The guidelines describe 4 main criteria, all of which should be met by designated authors. The 4 criteria are

contribution to project design or data acquisition and analysis;

drafting the work or critically revising the work;

final approval of the published work; and

accountability for ensuring that questions about the accuracy or integrity of any part of the work are appropriately investigated and resolved.

Although journal publishers often require authors to acknowledge that the first 3 criteria have been met, minimal mechanisms are in place to ensure that the guidelines are actually followed. Indeed, previous work has found that, even based on their own description of contributions cited at the end of papers, authors in as many as one third of publications failed to meet the ICMJE criteria ${ }^{2}$ and were deemed "honorary authors."

This high frequency of honorary authorship highlights a fundamental problem of lack of accountability in maintaining authorship standards. Furthermore, given that author self-report likely underrepresents the true frequency of the problem, we used an orthogonal and more unbiased approach to assess the consistency with which authorship standards are maintained. We investigated discordance in authorship between presentations of clinical trial data at American Society of Clinical Oncology (ASCO) annual meetings and the subsequent peer-reviewed publications. In particular, we focused our analysis on studies in which the number of study subjects in the clinical trial did not change between the Asco presentation and the final publication.

\section{METHODS}

Oral abstracts covering solid tumours presented at the 2015-2017 Asco annual meetings were identified $(n=560)$. The review was conducted from 19 September to 18 October 2018 and excluded non-industry-sponsored studies, nontherapeutic trials, duplicate listings, the absence of a subsequent peer-reviewed publication, a corresponding study publication preceding the Asco oral presentation, and studies in pediatric cancer ( $n=482$, Figure 1$)$. The remaining 78 abstract-publication pairs were examined to see if the same number of study participants were reported. The same number of study participants were reported in 52 abstract-publication pairs. We noted that the authors listed in the abstract-publication pair might be discordant because, for a given submission, Asco abstracts allow for 
only up to 20 authors. To account for that limit, we specifically examined authorship changes in the peer-reviewed publication when the abstract had fewer than 20 authors, leaving 35 abstract-publication pairs for the analysis.

\section{RESULTS}

Our assumption was that, with the same number of study subjects in both the abstract and the publication, it was unlikely that new authors appearing in the peer-reviewed publication contributed significantly enough to fulfil ICMJE criteria for authorship. However, in our analysis, we found that more than $70 \%$ of subsequent publications named additional authors not originally named on the abstract and that, on average, compared with the Asco oral abstract, papers gained a net of 2.5 authors at the time of publication (Table I). Interestingly, we also find that, for most studies, authors were removed or replaced at time of publication without clear explanation, further highlighting the discordance in authorship reporting.

To assess the possibility that increased time for additional data analysis could account for an increase in authors, we examined the interval between the Asco abstract submission and journal publication to see if a correlation between interval and authorship change was evident.

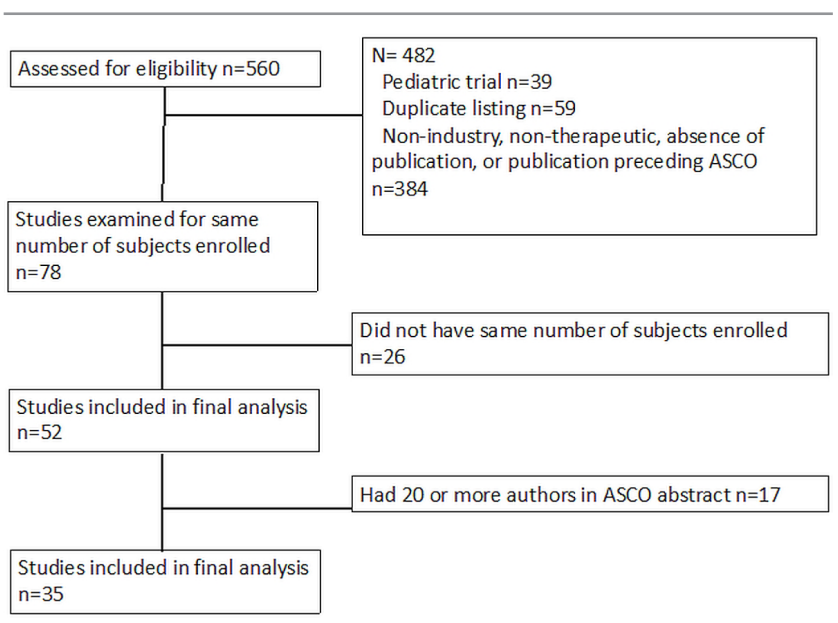

FIGURE 1 CONSORT diagram. ASCO = American Society of Clinical Oncology.
However, we found no significant correlation $(p=0.85)$ between the interval to publication and the author count.

\section{DISCUSSION AND CONCLUSIONS}

Overall, our analysis shows that $74.3 \%$ of Asco oral abstracts that result in peer-reviewed publications with no changes in total patients enrolled have authorship discordance suggestive of authorship changes that do not conform with ICMJE standards. Further, although it is certainly likely that some of those authorship changes do in fact conform with all 4 ICMJE criteria, the lack of transparency concerning the reasons that new authors are being added or removed is a direct reflection of the lack accountability in maintaining authorship standards. Much like recent financial conflicts of interest that have been garnering public scrutiny ${ }^{3-7}$, lack of some combination of uniformity, transparency, or compliance with established standards by peer-reviewed journals could explain the discordances. Potential solutions should focus on increased scrutiny and enforcement of consistent authorship reporting standards to foster a culture in which honorary authorship is unacceptable.

\section{CONFLICT OF INTEREST DISCLOSURES}

We have read and understood Current Oncology's policy on disclosing conflicts of interest, and we declare the following interests: GJW is currently an employee and stockholder of Unum Therapeutics; reports personal fees (ownership interest) from Circulogene and MiRanostics Consulting, and other personal fees from Paradigm, Ignyta, Spring Bank Pharmaceuticals, Pfizer, Viomics, Circulogene, IDEA Pharma, GLg Pharma, Angiex, Imaging Endpoints II, IBEx Medical Analytics, and Guidepoint Global; has received travel or accommodation support from Cambridge Healthtech Institute, GlaxoSmithKline, and Tesaro; and reports an issued patent PCT/ US2011/020612. DYG has no competing interests to declare.

\section{AUTHOR AFFILIATIONS}

*Harvard Medical School and ${ }^{\dagger}$ Beth Israel Deaconess Medical Center, Boston, MA, U.S.A.

\section{REFERENCES}

1. International Committee of Medical Journal Editors (ICMJE). Defining the Role of Authors and Contributors [Web resource]. n.l.: ICMJE; n.d. [Available at: http://www.icmje. org/recommendations/browse/roles-and-responsibilities/ defining-the-role-of-authors-and-contributors.html; cited 27 September 2019]

TABLE I Summary statistics of papers included in the analysis

\begin{tabular}{|c|c|c|c|c|}
\hline \multirow[t]{2}{*}{ Variable } & \multicolumn{4}{|c|}{ Year of abstract submission } \\
\hline & 2015 & 2016 & 2017 & Overall \\
\hline Papers that met criteria $(n)$ & 13 & 13 & 9 & 35 \\
\hline Papers with authors added [ $n(\%)]$ & $10(76.9)$ & $9(69.2)$ & $6(66.7)$ & $25(71.4)$ \\
\hline Authors added $(n)$, averaged & 3.6 & 3.9 & 1.6 & 3.2 \\
\hline Papers with authors removed $[n(\%)]$ & $7(53.9)$ & $5(38.5)$ & $3(33.3)$ & $15(42.9)$ \\
\hline Authors removed $(n)$, averaged & 1.3 & 0.4 & 0.3 & 0.7 \\
\hline Papers with any change $[n(\%)]$ & $10(76.9)$ & $10(76.9)$ & $6(66.7)$ & $26(74.3)$ \\
\hline Net change in author count $(n)$, averaged & 2.3 & 3.5 & 1.2 & 2.5 \\
\hline
\end{tabular}


2. Vera-Badillo FE, Napoleone M, Krzyzanowska MK, et al. Honorary and ghost authorship in reports of randomised clinical trials in oncology. Eur J Cancer 2016;66:1-8.

3. Tao DL, Boothby A, McLouth J, Prasad V. Financial conflicts of interest among hematologist-oncologists on Twitter. JAMA Intern Med 2017;177:425-7.

4. Kaestner V, Prasad V. Financial conflicts of interest among editorialists in high-impact journals. Blood Cancer J 2017;7:e611.

5. Johnston KL, Go RS. Financial conflicts of interest among ASCO annual meeting abstract authors, speakers, and planners. J Natl Cancer Inst 2007;99:1415-16.
6. Thomas K, Ornstein C. Top Sloan Kettering Cancer doctor resigns after failing to disclose industry ties [Web article]. The New York Times 2018; 13 September. [Available at: https://www.nytimes.com/2018/09/13/health/jose-baselga -cancer-memorial-sloan-kettering.html; cited 20 October 2018]

7. Vera-Badillo FE, Ocana A, Templeton AJ, Tibau A, Amir E, Tannock IF. Raising concern about the American Society of Clinical Oncology conflict of interest policy amendment. $J$ Clin Oncol 2014;32:3197. 